\title{
Granulomatose de wegener em paciente jovem - Relato de Caso
}

\author{
Granulomatosis of wegener in young patient- case report
}

Granulomatosis de wegener en paciente joven-relato de caso

\begin{abstract}
Carla Daniele Nascimento Pontes ${ }^{1 *}$, Yuri José Almeida da Silva ${ }^{1}$, Rodrigo Bona Maneschy ${ }^{1}$, Rudival Faial de Moraes Junior ${ }^{1}$, Marilia Vieira Miranda ${ }^{1}$, Lucianna Serfaty de Holanda ${ }^{1}$, Vitor Bruno Teixeira de Holanda ${ }^{1}$, Carolina Tavares Carvalho ${ }^{1}$, José Deorilo Cruz Gouveia Dos Santos Junior ${ }^{1}$, Milena Oliveira Saldanha1', Natalia Rodrigues Eugenio ${ }^{1}$, Henrique Lima de Sousa ${ }^{1}$.
\end{abstract}

\section{RESUMO}

Introdução: A Granulomatose de Wegener (GW), também conhecida como granulomatose com poliangeite, constitui uma doença autoimune rara e idiopática, que é marcada por induzir uma inflamação granulomatosa e vasculite sistêmica evolvendo vasos de pequeno e médio calibre. O diagnóstico precoce da GW exige um elevado índice de suspeição, pela baixa especificidade dos sintomas iniciais. Atualmente há uma escassez de dados relacionados ao perfil de pacientes no país, com suas características clínicas e epidemiológicas. Método: Estudo desenvolvido utilizando-se os dados do prontuário de atendimento do paciente, durante a internação. Descrição do relato de caso: Paciente do sexo masculino, 18 anos, foi admitido queixando-se de quadro de epistaxe, com início há duas semanas associado a Hipertensão Arterial Sistêmica (HAS) e oligúria. Evoluiu com quadro de síndrome urêmica e laboratório exibindo azotemia, sendo indicada diálise de urgência. Após investigação, apresentou C-ANCA reagente 1:20, sendo interrogado Granulomatose de Wegener pela presença do c-ANCA positivo, acometimento renal e sinusopatia/epistaxe. Evoluiu clinicamente sem novas intercorrências e recebeu alta hospitalar após liberação de vaga de diálise ambulatorial. Sendo encaminhado ao ambulatório de reumatologia. Conclusão: Torna-se necessário a inclusão da Granulomatose de Wegener na investigação etiológica de pacientes com história de insuficiência renal associada a acometimento de trato respiratório, dado a gravidade de evolução do quadro.

Palavras-chave: Granulomatose de Wegener, Trato Respiratório, Insuficiência Renal.

\begin{abstract}
Introduction: Wegener's granulomatosis (GW), also known as granulomatosis with polyangeitis, is a rare and idiopathic autoimmune disease, which is marked by inducing granulomatous inflammation and systemic vasculitis involving vessels of small and medium calibers. The early diagnosis of GW requires a high index of suspicion, due to the low specificity of the initial symptoms. Currently there is a shortage of data related to the profile of patients in the country, with its clinical and epidemiological characteristics. Method: This study was developed using data from the patient during a hospitalization period. Description of the case report: A male patient, 18 years old, was admitted complaining of epistaxis, starting two weeks ago with systemic arterial hypertension (SAH) and oliguria. He evolved with uremic syndrome and laboratory showing azotemia, being indicated emergency dialysis. After investigation, he presented a 1:20 c-ANCA reagent, Wegener's granulomatosis being interrogated for the presence of positive c-ANCA, renal involvement and sinusopathy / epistaxis. He progressed clinically without further complications and was discharged after ambulatory dialysis. Being referred to the rheumatology outpatient clinic. Conclusion: It is necessary to include Wegener's granulomatosis in the etiological investigation of patients with a history of renal insufficiency associated with respiratory tract involvement, given the severity of the disease progression.
\end{abstract}

Key words: Wegener's granulomatosis, Respiratory Tract, Renal Insufficiency.

${ }^{1}$ Fundação Santa Casa de Misericórdia do Pará, Belém- Pará. *E-mail: pontes4@live.com 


\section{RESUMEN}

Introducíon: La Granulomatosis de Wegener (GW), también conocida como granulomatosis con poliangeite, constituye una enfermedad autoinmune rara e idiopática, que está marcada por inducir una inflamación granulomatosa y vasculitis sistémica evolucionando vasos de pequeño y medio calibres. El diagnóstico precoz de GW requiere un alto índice de sospecha, por la baja especificidad de los síntomas iniciales. Actualmente hay una escasez de datos relacionados al perfil de pacientes en el país, con sus características clínicas y epidemiológicas. Método: Estudio desarrollado utilizando los datos del prontuario de atención del paciente durante la internación. Descripción del caso: El paciente de sexo masculino, de 18 años, fue admitido quejándose de cuadro de epistaxis, comenzando hace dos semanas asociado a Hipertensión Arterial Sistémica (HAS) y oliguria. Evolucionó con cuadro de síndrome urémico y laboratorio exhibiendo azotemia, siendo indicada diálisis de urgencia. Después de la investigación, presentó c-ANCA reactivo 1:20, siendo interrogado Granulomatosis de Wegener por la presencia del c-ANCA positivo, el acometimiento renal y la sinusopatía / epistaxis. Evolucionó clínicamente sin nuevas intercurrencias y recibió alta hospitalaria después de la liberación de vacante de diálisis ambulatorial. Siendo encaminado al ambulatorio de reumatología. Conclusión: Se hace necesario la inclusión de la Granulomatosis de Wegener en la investigación etiológica de pacientes con historia de insuficiencia renal asociada a acometimiento de tracto respiratorio, dado la gravedad de evolución del cuadro.

Palabras clave: Granulomatosis de Wegener, Trato Respiratorio, Insuficiencia Renal.

\section{INTRODUÇÃO}

A Granulomatose de Wegener (GW), também conhecida como granulomatose com poliangeite, constitui uma doença autoimune rara e idiopática, que é marcada por induzir uma inflamação granulomatosa e vasculite sistêmica evolvendo vasos de pequeno e médio calibres. (FERREIRA, 2011)

Apresenta uma incidência anual variando entre 2,1 e 15 casos por milhão de habitantes, em nível mundial. $\mathrm{E}$ acomete homens e mulheres na mesma proporção, com maior frequência em indivíduos na quinta década de vida. Clinicamente é subdividida em limitada e sistêmica. Sendo que, esta última, caracteriza - se pela ocorrência de envolvimento clínico de maior gravidade, incluindo o renal, que representa pior prognóstico (CAMPAINHA et al., 2013) (ANTUNES et al., 2005).

A granulomatose de Wegener é a vasculite que está mais associada à presença dos anticorpos anticitoplasma dos neutrófilos (ANCA), em número de casos. A presença de PR3 (principalmente com padrão c-ANCA) é observada em até $80 \%$ dos casos. Estudos têm mostrado que há correlação entre os títulos de ANCA e atividade da doença, além da frequência de recidivas durante o tratamento (FINKIELMAN et al., 2007; RIBEIRO et al., 2006). A causa exata da doença não foi identificada, e pode obedecer a uma predisposição genética, com acometimento de genes específicos, adicionado a fatores ambientais, infecciosos, de toxicidade e farmacológicos que gerariam o processo da doença (MORILLO, 2010).

Caracteriza-se clinicamente por acometer o trato respiratório superior principalmente com presença de úlceras mucosas e rinite inflamatória, além de epistaxes e lesão de cartilagem nasal e inferior com pneumopatias, tosse e hemoptise, além de sistema renal com glomerulonefrite, constituindo a sua tríade básica. Mas a GW também pode acometer o sitema ocular com inflamação orbitária, perda visual, visão dupla, conjuntivitem esclerite e episclerite. (FERNANDES et al., 2004).

O diagnóstico precoce da GW exige um elevado índice de suspeição, pela baixa especificidade dos sintomas iniciais. Por esta razão é por vezes diagnosticado apenas após envolvimento multiorgânico ou na presença de refratariedade do problema inicial à terapêutica (DIOGO et al., 2011).

A granulomatose de Wegener pode manifestar-se com diferentes graus de gravidade, requerendo por vezes, tratamento imunosupressor agressivo e plasmaférese. Dessa maneira, cada caso deve ser analisado e conduzido de forma específica, de acordo com os riscos e benefícios para o paciente, com o intuito de evitar complicações mais severas da doença (RIBEIRO et al., 2006). 
Atualmente há uma escassez de dados relacionados ao perfil de pacientes no país, com suas características clínicas e epidemiológicas. Necessitando a realização de novas pesquisas de âmbito nacional, com o objetivo de traçar a prevalência dessas doenças raras no Brasil e em suas regiões. Facilitando um diagnóstico e uma abordagem terapêutica precoce, além de um seguimento clínico mais padronizado.

Portanto, o presente estudo se propõe a realizar uma revisão de literatura baseada em um relato de caso de um paciente jovem, internado em um Hospital Universitário no Estado do Pará, com diagnóstico de Granulomatose de Wegener. E assim, contribuir com a fundamentação do manejo terapêutico desses quadros e incentivar novas publicações sobre o tema.

\section{RELATO DE CASO}

No ano de 2017, na Fundação Santa Casa de Misericórdia no Pará (FSCMPA), ocorreu um caso de um paciente jovem, hospitalizado com diagnóstico de Granulomatose de Wegener.

No dia 28 de junho de 2017, paciente do sexo masculino, 18 anos, foi admitido no setor de Clínica Médica da FSCMPA, queixando-se de quadro de epistaxe, com início há duas semanas. Associado a Hipertensão Arterial Sistêmica (HAS), com pico hipertensivo máximo de $180 \times 100 \mathrm{mmHg}$ e oligúria. Negava outros sintomas ou desenvolvimento de edema durante evolução e antecedentes mórbidos pessoais, até o momento.

Ao exame físico, apresentava-se hipocorado ++/4+, febril $(37,4 \stackrel{\circ}{\circ})$, sopro sistólico panfocal, além de hepatomegalia de aproximadamente $3 \mathrm{~cm}$ abaixo do rebordo costal. Em relação aos exames laboratoriais da admissão, possuía hemograma com anemia (hemoglobina: 6,2 g/dl- Referência: 12 a $18 \mathrm{~g} / \mathrm{dl}$ ), plaquetopenia (51.170/mm3- Referência: 150.000 a $440.000 / \mathrm{mm} 3$ ), leucocitose com desvio a esquerda (leucócitos: 15.160/mm3- Referência: 4.000 a 10.000/mm3) e alteração da função renal (creatinina: $13,1 \mathrm{mg} / \mathrm{dL}$ Referência: até $1,3 \mathrm{mg} / \mathrm{dL}$ e ureia: $391 \mathrm{mg} / \mathrm{dL}$ - Referência: 17 a $43 \mathrm{mg} / \mathrm{dL}$ ). Apresentando outros resultados como urinálise de coloração amarelada, ligeiramente turva, hemoglobina +++, glicose +, linfócitos: 20-30 por campo, hemácias: 40-50 por campo e cristais: uratos amorfos frequentes. Sendo iniciado antibioticoterapia com Ceftriaxona ( $2 \mathrm{~g} / \mathrm{dia}$ ) devido quadro infeccioso.

Devido quadro de síndrome urêmica e laboratório exibindo azotemia, o paciente foi avaliado pela nefrologia e submetido a implante de cateter duplo lúmen (CDL) em femoral direita para realização de diálise de urgência. Sendo posteriormente solicitado proteinúria de $24 \mathrm{~h}$, com resultado de $969 \mathrm{mg} / 24$ horas (volume urinário: $1.700 \mathrm{ml})$.

Simultaneamente foi solicitado acompanhamento com a equipe da hematologia para investigação desse padrão de bicitopenia, além de outros exames laboratoriais, sorologias, marcadores imunológicos, endocrinológicos e relacionados ao padrão hematológico do paciente.

No sétimo dia de internação (04/07/2017), o paciente apresentou novo episódio de epistaxe com melhora após tampão com duas ampolas endovenosa de adrenalina $(1 \mathrm{mg} / \mathrm{ml})$ e de ácido tranexâmico $(50 \mathrm{mg} / \mathrm{ml})$. Além de leve hiperemia conjuntival, principalmente à direita. Após estabilização clínica, foi realizada tomografia computadorizada de seios da face, tendo como resultado o espessamento mucoso dos seios maxilar, etmoidal e esfeinodal à esquerda, com obstrução dos complexos ostiomeatais correspondentes. Presença de cisto de retenção na parede medial do seio maxilar esquerdo e células da mastoide pouco areadas e veladas bilateralmente.

Neste mesmo dia, o paciente realizou ecocardiograma apresentando aumento das áreas cardíacas, fração de ejeção do ventrículo esquerdo (FEVE): 70\%, importante grau de hipertrofia miocárdica simétrica do ventrículo esquerdo (VE). Além de desempenho global aumentado (hiperdinâmico) em VE, valvas cardíacas e pericárdio sem alterações.

Em relação aos exames laboratoriais, as sorologias encontravam-se não reagentes para HIV, Hepatites B e C, Sífilis e Toxoplasmose IgM. E os marcadores imunológicos negativos para Lúpus Eritematoso Sistêmico. 
No décimo dia de internação (07/07/2018), foi coletado mielograma para elucidar permanência da plaquetopenia associada à anemia, sugerindo hipoplasia medular inespecífica.

No dia 12/07/2017 foi realizada ultrassonografia (usg) de rins e vias urinárias, demonstrando rim direito com diferenciação corticomedular preservada, porém se detecta acentuação da ecogenecidade cortical, sugerindo nefropatia parenquimatosa aguda/subaguda.

A reumatologia sugeriu presença de vasculite, indicando pesquisa do anticorpo-ANCA e anticardiolipinas, além de iniciar corticoterapia com prednisona $1 \mathrm{mg} / \mathrm{kg} / \mathrm{dia}(50 \mathrm{mg} / \mathrm{dia})$ e observar resposta clínica. A biópsia renal sugerida foi impossibilitada devido descompensação dos seus níveis pressóricos e pela manutenção da plaquetopenia.

Foi obtido resultado positivo para c-ANCA (1:20) e Anti cardiolipina IgM, sendo então diagnosticado com Granulomatose de Wegener, pela presença concomitante do acometimento renal e sinusopatia/epistaxe. Aumentando dessa maneira a dose de prednisona para $2 \mathrm{mg} / \mathrm{kg} / \mathrm{dia}-100 \mathrm{mg} / \mathrm{dia}$.

Posteriormente realizou-se pulsoterapia com metilprednisona 1g/dia por três dias, com profilaxia prévia para estrongiloidíase com anti-helmíntico. Após término, a dose de prednisona foi reajustada para $60 \mathrm{mg} / \mathrm{dia}$.

Evoluindo satisfatoriamente bem clinicamente e com liberação da terapia renal substitutiva, teve alta hospitalar programada para o dia 06/09/2017. Sendo encaminhado ao ambulatório de reumatologia e confirmada vaga de diálise ambulatorial em Ulianópolis. Manteve como medicações: hidralazina 50mg 6/6 horas; nifedipino $20 \mathrm{mg} 8 / 8$ horas; metildopa $500 \mathrm{mg} 8 / 8$ horas; carvedilol 12,5mg 12/12 horas; prednisona $60 \mathrm{mg} / \mathrm{dia}$; omeprazol 20mg/dia e complexo B.

\section{DISCUSSÃO}

A granulomatose de Wegener (GW) apresenta um amplo espectro de manifestações e continua a ser um dos diagnósticos mais desafiadores na prática clínica. Pois apesar dos sintomas respiratórios e renais mais comuns, pode também se desenvolver a partir de complicações menos frequentes. Dessa forma, torna-se difícil a distinção de outras etiologias e até mesmo de queixas isoladas (MORILLO, 2010).

Essa doença corresponde a uma vasculite necrosante e granulomatosa de origem idiopática, que acomete pequenas e médias artérias das vias respiratórias altas/baixas e rins. Apresenta prevalência estimada em 3:100.000 habitantes e pico de incidência entre a quarta e quintas décadas de vida (SANTOS et al., 2009).

Mesmo com a possibilidade de acometer todas as faixas etárias, a GW raramente desenvolve-se em indivíduos mais jovens, como observado no presente estudo, no qual o paciente apresentava 18 anos quando iniciou os primeiros sintomas.

As grandes séries demonstram uma quantidade de envolvimento semelhante em ambos os gêneros (ERICKSON e HWANG, 2007). Não sendo evidenciada na literatura amostras com diferença importante no predomínio entre o sexo masculino e feminino.

As manifestações sistêmicas da GW são muito variadas, caracterizando-se pela tríade de inflamação granulomatosa das vias aéreas altas e baixas, vasculite necrotizante com PR3 -ANCA positivos e glomerulonefrite. Os sintomas do trato respiratório são os mais comuns (50-85\% dos casos) e representam, na maioria das vezes, o quadro inicial da doença. Sendo incluída, a obstrução nasal, rinorreia (purulenta/ sanguinolenta), epistaxe, sinusite, otite média, nariz em sela por perfuração do septo nasal; tosse, hemoptise, dispneia, dor torácica (pleurítica); estridor por estenose subglótica ou traqueobrônquica (REIS, 2012).

O início dos sintomas no caso apresentado foi desencadeado por um sintoma respiratório, pela presença de epistaxe, concordando com estudos anteriores. E simultaneamente teve associação com acometimento renal, devido quadro de insuficiência e azotemia. Segundo Cairoli et al. (2008), o envolvimento renal aparece aproximadamente em $80 \%$ dos casos, manifestando-se por proteinúria, hematúria e insuficiência renal. As manifestações histopatológicas vão desde formas leves, como a glomerulonefrite segmentar e focal, a glomerulonefrite rapidamente progressiva, que frequentemente cursa com doença renal dialítica. 
A presença de alterações cutâneas está presente em aproximadamente $45 \%$ dos pacientes, podendo manifestar-se como úlceras necróticas, púrpuras palpáveis ou por outras formas. $\mathrm{O}$ acometimento mucoso também pode ser representado pelas úlceras orais e nasais, além da gengivite. (PEREIRA et al., 2007; BRANDT et al., 2009). Todavia, não foi observado envolvimento dermatológico no caso analisado.

No presente estudo, o paciente apresentava alterações clínicas e estruturais relacionadas a sintomas cardiovasculares. Com presença de sopro sistólico panfocal, além de ecocardiograma com aumento das áreas cardíacas e importante grau de hipertrofia miocárdica. Estes achados são considerados incomuns, com frequência de aproximadamente $15 \%$ dos casos. Sendo ocasionados pela vasculite e efeitos dos granulomas, desenvolvendo-se na forma de pericardite, miocardite, doença valvar, insuficiência cardíaca ou alterações do sistema de condução (SANTOS et al., 2009; PATIÑO, 2016). As manifestações oculares são consideradas ainda menos prevalentes, encontrada em apenas 10\% dos pacientes. Com desenvolvimento de conjuntivite, uveíte, ptose palpebral, pseudotumor retro-orbitário, neurite óptica ou até episódios de hiperemia conjuntival, como ocorreu durante a evolução do caso. Porém, sem demais repercussões clínicas graves ou progressão de piora (MORILLO, 2010).

A análise laboratorial complementa a avaliação clínica no estabelecimento do diagnóstico e permite monitorizar a evolução da doença, a efetividade do tratamento, bem como os seus potenciais efeitos tóxicos. O estudo inicial deve incluir hemograma, marcadores inflamatórios, estudo da função hepática e renal, exame da urina e estudo imunológico (MACHADO, 2013). De acordo com o American College of Rheumatology $(\mathrm{ACR})$, a presença de dois ou mais desses critérios fornecem o diagnostico final da granulomatose de Wegener: Inflamação oral ou nasal (úlceras orais/nasais ou descarga purulenta/sanguinolenta); Radiografia de tórax, com lesões nodulares, infiltrados fixos ou cavitações pulmonares; Sedimento urinário, com hematúria micro ou macroscópica ou cilindros hemáticos; Biópsia de órgãos acometidos, como, seios da face, mucosa de vias aéreas superiores, rins e pulmões. (FERREIRA, 2011).

A presença de anticorpos anti-citoplasma de neutrófilos (c-ANCA) são marcadores essenciais e auxiliam no diagnóstico da doença, pois possuem alta especificidade e encontram-se positivos em aproximadamente 70-97\% dos casos. Todavia, a biópsia representa papel de destaque no seu diagnóstico etiológico. Ocorrendo o envolvimento de arteríolas e vênulas de pequeno calibre, podendo ser necrotizante ou granulomatosa, com infiltrado inflamatório misto e padrão geográfico de necrose tecidual (PEREIRA et al., 2007; RIBEIRO et al., 2006).

O paciente apresentou como critérios diagnósticos a presença de inflamação nasal com epistaxe e tomografia dos seios da face com espessamento mucoso dos seios maxilar, etmoidal e esfeinodal à esquerda, com obstrução dos complexos ostiomeatais correspondentes e hematúria microscópica no exame admissional. Outros sintomas e marcadores auxiliaram na confirmação da doença, como a positividade do ANCA de padrão citoplasmático e desenvolvimento de doença renal. A biópsia a nível hospitalar não foi realizada devido instabilidade clínica inicial e posteriormente pelos baixos níveis plaquetários do paciente.

A base terapêutica fundamenta-se na introdução de corticosteroides e imunossupressores precocemente. Pois, sem tratamento, $90 \%$ dos pacientes apresentam elevados índices de mortalidade em até dois anos do início dos sintomas, devido ao desenvolvimento de uremia ou insuficiência respiratória (BRANDT et al., 2009). A abordagem é dividida em terapia de indução e de manutenção. Sendo a primeira prescrita para as formas ativas, tanto no início quanto em recidivas da doença. Seu objetivo é alcançar a remissão completa e evitar acúmulos de danos (SBR, 2017).

Assim recomenda-se o emprego de glicorticóides sistêmicos, com uso de prednisona em dose inicial de 0,5-1 $\mathrm{mg} / \mathrm{kg} / \mathrm{dia}$ por uma a quatro semanas, seguida por sua redução lenta e gradativa. Essa terapia deve durar pelo menos seis meses e em alguns casos prolongando-se até dois anos. Em pacientes com envolvimento grave pode-se optar por receber previamente três doses intravenosas de metilprednisolona, retornando posteriormente com a dose oral inicial (SBR, 2017; MORILLO, 2010; ANTUNES et al., 2005).

Devido aperfeiçoamento no diagnóstico e estratégias terapêuticas, a GW evoluiu com um melhor prognóstico nos últimos quarenta anos, com redução nas taxas de recidiva e morbidade. Porém, ainda há um 
índice de mortalidade geral de aproximadamente 12,5 -25,7\%. E os principais contribuintes para isso, incluem vasculite ativa, doença cardiovascular, malignidade e mais comumente quadros infecciosos (GRYGIELGÓRNIAK et al., 2018).

Além desses citados, pacientes que necessitam de diálise no início da doença ou ao diagnóstico anunciam pior progressão clínica. Com sobrevida renal média entre 2 e 5 anos, de $86 \%$ e 75\%, respectivamente, com provável desenvolvimento de insuficiência renal crônica terminal (RIBEIRO et al., 2006).

O relato estudado demonstra que a granulomatose de Wegener é um acometimento clínico raro que necessita de uma investigação etiológica precoce nos primeiros sintomas. Buscando um diagnóstico amplo que associe suas diversas manifestações, e assim visando uma melhor abordagem terapêutica e maior sobrevida para esses pacientes.

\section{REFERÊNCIAS}

1. ANTUNES T, BARBAS CSV. Granulomatose de Wegner. Jornal Brasileiro de Pneumologia, 2005; 31: S21S6.

2. BRANDT HRC, ARNONE M, VALENTE NYS, et al. Vasculites dos médios e grandes vasos. An Bras Dermatol, 2009; 84(1): 57-67.

3. CAMPAINHA S, GONÇALVES M, TAVARES P, et al. Granulomatose com poliangeíte inicialmente diagnosticada como cancro do pulmão. Rev Port Pneumol,2013; 19(1): 45-48.

4. CAIROLI DE SILVARIÑO R, MENDEZ E. Granulomatosis de Wegener: clínica, diagnóstico y tratamiento a propósito de cinco casos. Rev Med Uruguay, 2008; 24: 37-41.

5. DIOGO CJ, FORMIGO A, FLOROVA E, et al. Granulomatose de Wegener. Relato de casos. Rev Bras Clin Med, 2011; 9(6): 437-440.

6. ERICKSON VR, HWANG PH. Wegener's granulomatosis: current trends in diagnosis and management. Curr Opin Otolaryngol Head Neck Surg, 2007; 15: 170-176.

7. FERNANDES NC, MICAELO LF, CUZZI T. Granulomatose de Wegener localizada. Med Cutan Iber Lat Am, 2004; 32(5): 218-222.

8. FERREIRA MCF, JUNIOR HP. Granulomatose de Wegener. Relato de caso. Revista Brasileira de Clínica Médica, 2011; 9(5): 389-392.

9. FINKIELMAN JD, MERKEL PA, SCHROEDER D, et al. Antiproteinase 3 antineutrophil cytoplasmic antibodies and disease activity in Wegener granulomatosis. Ann Intern Med, 2007; 6(147): 611-619.

10. GRYGIEL-GÓRNIAK B, LIMPHAIBOOL N, PERKOWSKA, K.; PUSZCZEWICZ, M. Clinical manifestations of granulomatosis with polyangiitis: key considerations and major features. Postgraduate medicine, 2018.

11. MACHADO FCF.Vasculites - Avanços diagnósticos e terapêuticos. Tese de Mestrado. Faculdade de Medicina- Universidade do Porto, 2014; 42p.

12. MORILLO MM. Granulomatosis de Wegener estudio de 15 casos, supervivencia y dañocrônico. Trabayo de investigacion, Universitat Autònoma de Barcelona, 2010; 64p.

13. PATIÑO, S.H. Granulomatosis con poliangeítis: actualización y: conceptos claves. Revista Cubana de Reumatología, 2016; 18(1): 36-44.

14. PEREIRA CI, ZACHARIAS LC, ZAGUI R et al. Granulomatose de Wegener: relatos de casos. Arq Bras Oftalmol, 2007; 70 (6): 1010-1015.

15. REIS MG. Pulmão e doenças sistêmicas: Vasculites. Revista de Pediatria do Centro Hospitalar do Porto, 2012; 21(3): S184-188.

16. RIBEIRO C, NETO MSCN, SILVA GMC et al. Granulomatose de Wegener: Apresentação Clínica e Tratamento. J Bras Nefrol, 2006; 28(2): 114-117.

17. SANTOS A, SACFI ACB, MORAIS LA et al. Apresentação e Evolução Atípicas da Granulomatose de Wegener: Relato de Caso. Revista Ciências em Saúde, 2015; 5(3).

18. SOCIEDADE BRASILEIRA DE REUMATOLOGIA. Recomendações da Sociedade Brasileira de Reumatologia para a terapia de indução para vasculite associada à ANCA. Revista Brasileira de Reumatologia, 2017; 57(2): S484-496. 